
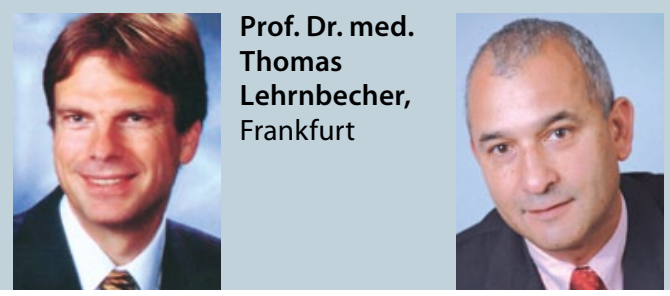

Prof. Dr.
Tino F.
Schwarz,
Würzburg

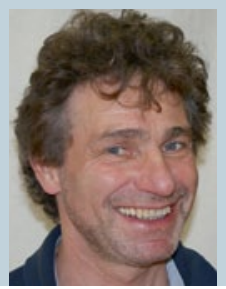

Dr.

Thomas

Spindler,

Wangen

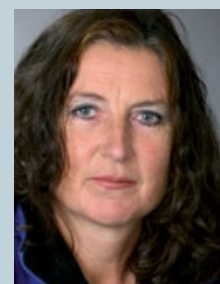

Dr. med.

Kirsten

Stollhoff,

Hamburg

\title{
Asthmatiker sind häufig noch Zigarettenrauch ausgesetzt
}

\section{Tabakrauchexposition führt bei Kindern und Jugendlichen mit Asthma bekanntermaßen zu einer erhöhten Morbidität. Eine amerikanische Studie zeigt, dass es seit den 90er-Jahren zwar zu einem Rückgang der Exposition von asthmakranken Kindern und Jugendlichen gekommen ist, die Mehrzahl jedoch weiterhin in der häuslichen Umgebung Tabakrauch ausgesetzt ist.}

m amerikanischen „National Health and Nutrition Examination Survey“ (NHANES) wurden Daten von 2.250 asthmakranken Kindern und Jugendlichen zwischen vier und 19 Jahren in den Zeiträumen 1988-1994, 1999-2004 und 2005-2010 ausgewertet. OutcomeParameter waren: der Gebrauch von Tabakprodukten (Zigaretten, Zigarren und Pfeifen) anhand eines Cotininwertes im Serum von $>10 \mathrm{ng} / \mathrm{ml}$ oder einer entsprechenden Eigenanamnese und, unter Nichtrauchern, die Tabakrauchexposition (Cotininwert $\geq 0,05 \mathrm{ng} / \mathrm{ml}$ und Nikotingebrauch in der häuslichen Umgebung). Untersucht wurde die Beziehung zwischen den Outcome-Parametern und Alter, Geschlecht, ethnischer Herkunft sowie Familieneinkommen.

Brian Kit und Kollegen zeigten, dass zwischen 2005 und 2010 noch 17,3\% der asthmakranken Jugendlichen zwischen zwölf und 19 Jahren selbst Tabakprodukte konsumierten. Von den Kindern und Jugendlichen zwischen vier und 19 Jahren, die selbst nicht rauchten, waren $53,2 \%$ regelmäßig Tabakrauch exponiert, $17,3 \%$ in der direkten häuslichen Umgebung. Unter den Familien mit niedrigem Einkommen betrug die Rate sogar 70,1\%, bzw. 28,1\% in Bezug auf die häusliche Umgebung. Die soziodemographischen Daten zeigten auch, dass bei den Jugendlichen mit niedrigem Einkommen die Prävalenz bzgl. Tabak-

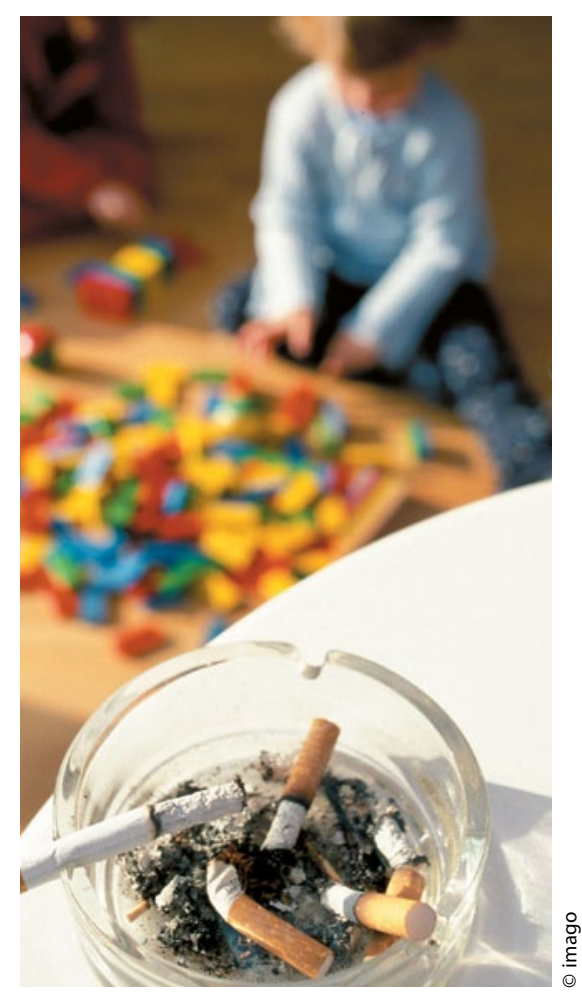

Kinder mit Asthma bronchiale rauchen nach wie vor häufig passiv mit. rauchexposition und häuslichen Nikotingebrauch in den drei Perioden persistierte. Insgesamt kam es zwischen 19881994 und 2005-2010 aber zu einem Rückgang bei beiden Parametern. In Bevölkerungsgruppen mit geringem Einkommen war dieser Trend aber nicht statistisch signifikant.

Kit BK et al. US prevalence and trends in tobacco smoke exposure among children and adolescents with asthma. Pediatrics 2013; 131: 407-14

Kommentar: Trotz eines Rückganges des aktiven Tabakgebrauchs und auch der passiven Tabakrauchexposition von Kindern und Jugendlichen mit Asthma in den letzten 20 Jahren ist weiterhin die Mehrzahl der amerikanischen pädiatrischen Asthmapatienten dieser schädlichen Substanz regelmäßig ausgesetzt.

Es besteht eine klare Abhängigkeit zwischen aktiver und passiver Nikotinexposition und sozialem Status. Auch wenn es sich hier um amerikanische Daten handelt, besteht sicherlich eine gewisse Übertragbarkeit auf die deutschen Verhältnisse. Insbesondere in Hinblick auf die nachgewiesene Wirksamkeit eines konsequenten Nichtraucherschutzes mit Rückgang der Hospitalisierungsrate atemwegskranker Kinder [Mackay D et al. N Engl J Med 2010; 363: 1139-45], aber auch mit Rückgang der Krankenhausaufnahmen wegen Herzbeschwerden bei Erwachsenen in Deutschland (DAK-Studie 2012) sind weitere, durchgreifende Maßnahmen zum Schutz unserer Bevölkerung und insbesondere der Kinder und Jugendlichen zu fordern. Besondere Bemühungen sollten in die Aufklärung und Hilfestellung für sozial schwache Bevölkerungsgruppen gesteckt werden.

Dr. Thomas Spindler 